\title{
Hem-o-lok Clip-Related Complications After Colorectal Surgery
}

\author{
loannis Nikolopoulos, MRCS, Janakan Ghananandan, MRCS, Rajab Kerwat, FRCS \\ Queen Elizabeth Hospital, London, United Kingdom (all authors).
}

\begin{abstract}
Introduction: Hem-o-lok ligation clips are widely used for the hemostatic control of major mesenteric vessels during laparoscopic colorectal resection. We report 2 rare cases of bowel obstruction and perforation requiring urgent surgical intervention after the application of Hem-o-lok clips (Weck Surgical Instruments, Teleflex Medical, Durham, NC, USA), which were used to occlude vessels and provide hemostasis during elective laparoscopic sigmoid colon resection. In both cases, the clips completely dislodged from the vessels to which they were initially applied and eventually attached to the wall of the small bowel, causing obstruction, ischemia, and perforation.
\end{abstract}

Case Descriptions: Case 1. An 82-year-old man with Duke's $\mathrm{C}$ adenocarcinoma underwent an elective laparoscopic reversal of Hartmann's procedure after an elective laparoscopic sigmoid colectomy, which was converted to a Hartmann's procedure due to an anastomotic leak. The reversal of the Hartmann's operation was complicated by dense adhesions of small bowel to the inferior mesenteric artery (IMA) remnant, with the Hem-o-lok clips initially used to occlude the artery embedded in the wall of the small bowel and completely dissociated from the IMA. A perforation occurred, associated with the clip-related stricture of the small bowel. Case 2. A 69-year-old woman underwent an urgent laparotomy for a small-bowel obstruction that did not respond to conservative treatment. Her surgical history of perforated diverticular disease included a laparoscopic Hartmann's procedure that was reversed laparoscopically a few months later. She was found to have a small-bowel obstruction secondary to a Hem-o-lok clip that was completely embedded in the serosa of the bowel wall, with a perforation just proximal to it.

Conclusion: Ligation clips, when properly applied, are regarded as a reliable method of obtaining arterial hemostatic control during laparoscopic colorectal procedures. Migration of the clips, although a rare problem, can cause serious complications that may necessitate further surgical intervention. Previous case reports have shown that Hem-o-lok clips can cause vascular complications. These two cases illustrate that they can cause bowel-related complications that necessitate emergent surgical treatment. It is therefore our view that these clips should not be used for arterial ligation during laparoscopic colorectal surgery.

Key Words: Bowel obstruction, Hem-o-lok clip, Laparoscopic colorectal surgery, Perforation.

Citation Nikolopoulos I, Ghananandan J, Kerwat R. Hem-o-lok clip-related complications after colorectal surgery. CRSLS e2014.00022. DOI: 10.4293/JSLS.2014.00022.

Copyright ( 2015 SLS This is an open-access article distributed under the terms of the Creative Commons Attribution-Noncommercial-ShareAlike 3.0 Unported license, which permits unrestricted noncommercial use, distribution, and reproduction in any medium, provided the original author and source are credited.

Address correspondence to: Ioannis Nikolopoulos, MB BS, MSc, Queen Elizabeth Hospital, Stadium Road, London SE18 4QH, UK. Telephone: 020-8836-5488, Fax 020-8836-5436, E-mail: inikolopoulos@gmail.com

\section{INTRODUCTION}

Hem-o-lok clips (Weck Surgical Instruments, Teleflex Medical, Durham, NC, USA) have been used for vascular control in urologic, gynecologic, general, and colorectal laparoscopic surgery. Some studies have suggested that, when applied properly, the Hem-o-lok clip is a safe method of achieving vascular control and hemostasis, ${ }^{1}$ especially during laparoscopic nephrectomy. ${ }^{2}$ Other stud- ies have demonstrated the safety of these clips in ligating the appendiceal stump ${ }^{3,4}$ and have advocated its use in hysterectomy, ${ }^{5}$ prostatectomy, ${ }^{6}$ and lobectomy. ${ }^{7}$ The Food and Drug Administration (FDA), however, issued a warning to health care providers that Hem-o-lok clips can pose a serious risk to the donor during a laparoscopic living-donor nephrectomy when used for the ligation and occlusion of the renal artery. ${ }^{8}$ The warning followed 15 reports of 12 injuries and 3 deaths that occurred between 
November 2001 and March 2005..$^{8}$ In 2006, the manufacturer amended the information in the instructions for the use of Hem-o-lok clips. ${ }^{9}$ Since then there have been reports of cases of clip-related complications after robotassisted laparoscopic radical prostatectomy, ${ }^{10,11,12}$ including small-bowel obstruction, ${ }^{13}$ laparoscopic radical nephroureterectomy with bladder cuff excision, ${ }^{14}$ and laparoscopic cholecystectomy. ${ }^{15}$ We report 2 cases of small-bowel obstruction and perforation caused by Hemo-lok clips initially applied for the occlusion of the inferior mesenteric artery (IMA), during elective laparoscopic sigmoid colon resection.

\section{CASE REPORTS}

\section{Case 1}

An 82-year-old man underwent an elective laparoscopic sigmoid colectomy for a Duke's C moderately differentiated adenocarcinoma. After surgery, an anastomotic leak developed that led to an urgent laparotomy and the formation of an end colostomy. After discharge from the hospital, the patient successfully completed 8 cycles of adjuvant chemotherapy.

He then underwent a laparoscopic reversal of the Hartmann's procedure. He was found to have very dense small-bowel adhesions to the area that corresponded to the origin of the IMA, with 2 Hem-o-lok clips embedded in the small-bowel serosa and completely dislodged from the IMA remnant to which they had initially been applied for hemostasis (Figure 1). There was no active bleeding



Figure 1. A Hem-o-lok clip embedded in the serosa of the bowel wall. from the artery. After division of adhesions, there was a small-bowel perforation next to one of the Hem-o-lok clips completely walled off by the numerous adhesions, which led to resection of the affected small-bowel loop and a stapled side-to-side anastomosis.

Unfortunately, the patient had a complicated recovery from the operation, as he underwent an urgent laparotomy for small-bowel perforation, and he was found to have a leak from the area of the previously repaired enterotomy.

\section{Case 2}

A 69-year-old woman presented with a 2-day history of lower abdominal pain, vomiting, and absolute constipation. Her previous surgical history included an emergency laparoscopic Hartmann's procedure 14 months earlier for perforated sigmoid diverticulitis, with a laparoscopic reversal of Hartmann's procedure 8 months later.

On examination, she had generalized abdominal tenderness, distension, and absent bowel sounds. Initial blood tests revealed a white blood cell count of $20.1 \times 10^{9} / \mathrm{L}$ and a C reactive protein level of $223 \mathrm{mg} / \mathrm{L}$. A plain abdominal $\mathrm{x}$-ray (Figure 2) revealed a small-bowel obstruction, and a computed tomographic scan of the abdomen and pelvis (Figure 3) showed markedly dilated loops of small bowel, particularly in the left flank, but an obvious cutoff point of obstruction.

The obstruction was initially treated conservatively, as it was thought to be adhesional, but as her symptoms had not resolved by day 4 , she proceeded to an emergency laparotomy. Intra-operative findings were dilated smallbowel loops with a cutoff point. At the transition point, a Hem-o-lok clip was embedded in the serosa of the small bowel, causing secondary stenosis. Just proximal to this area, there was a small-bowel perforation (Figure 4). The affected small bowel was resected, and a double-barreled ileostomy was formed. The Hem-o-lok clip had been placed for ligation of the IMA during the laparoscopic Hartmann's procedure.

\section{DISCUSSION}

Hem-o-lok clips (Weck Surgical Instruments, Teleflex Medical, Durham, NC, USA) are made from a permanent radiotranslucent inert polymer. They are available in 4 sizes and are designed to ligate vessels from 2 to $16 \mathrm{~mm}$. There have been reports supporting the safety, ease of application, and cost effectiveness of the clips in several general surgery procedures such as laparoscopic appen- 


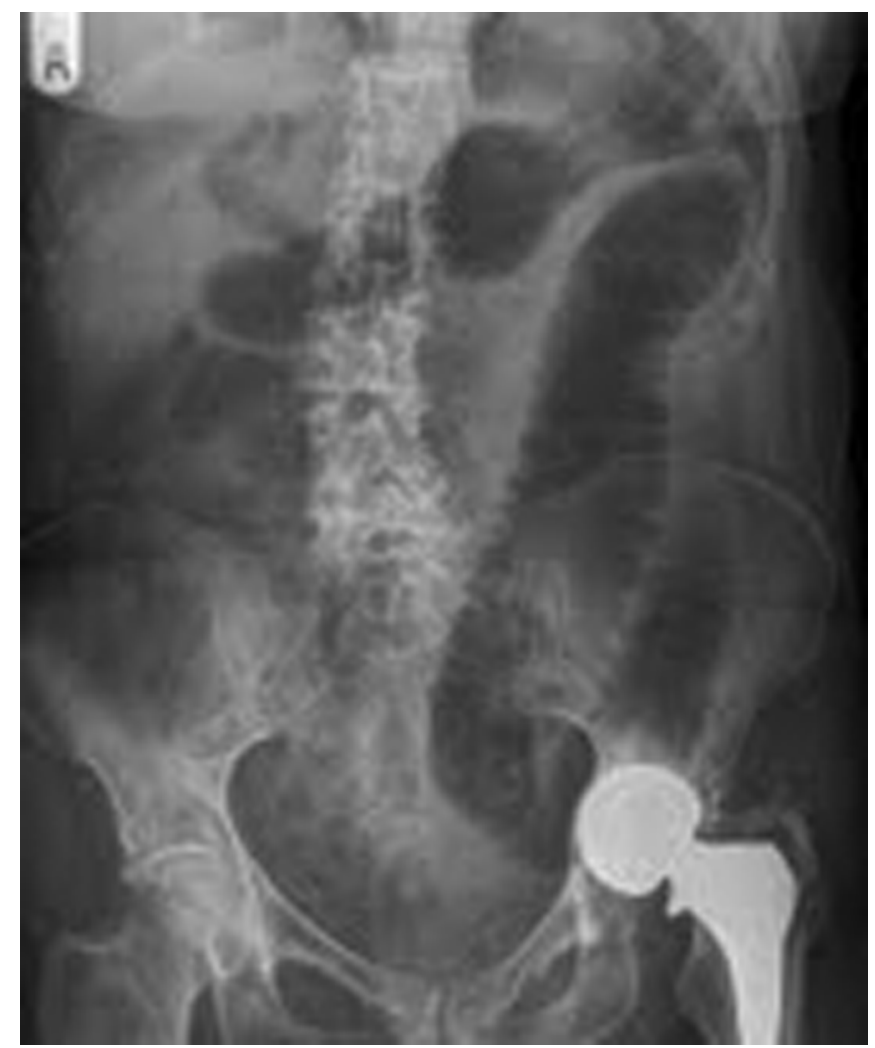

Figure 2. Plain abdominal x-ray showing a small bowel obstruction.

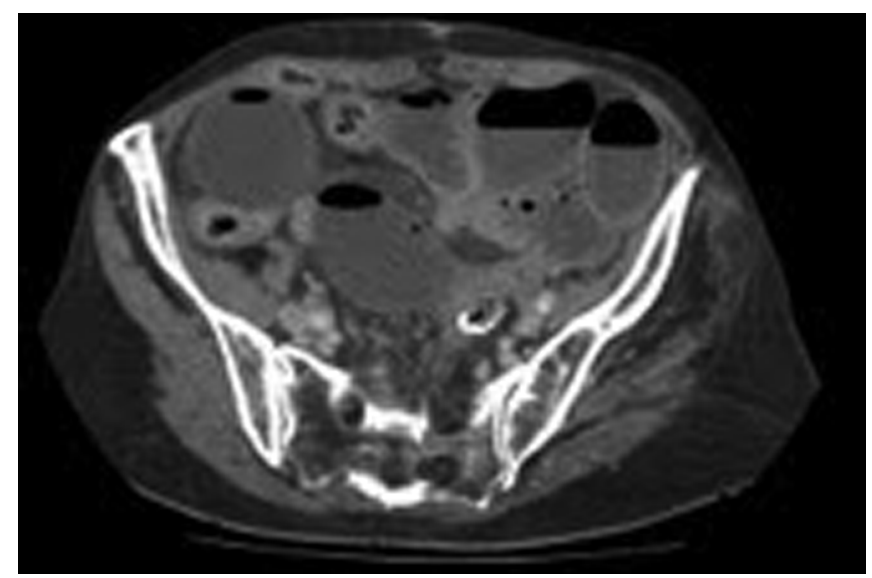

Figure 3. Computed tomographic scan of the small bowel.

dectomy and cholecystectomy, when appropriate-size clips are applied at the appendicular stump and artery, as well as the cystic duct and artery. A retrospective review ${ }^{1}$ of a single center's experience with the use of Hem-o-lok clips during laparoscopic appendectomy, cholecystectomy, and splenectomy did not show any clip failure or

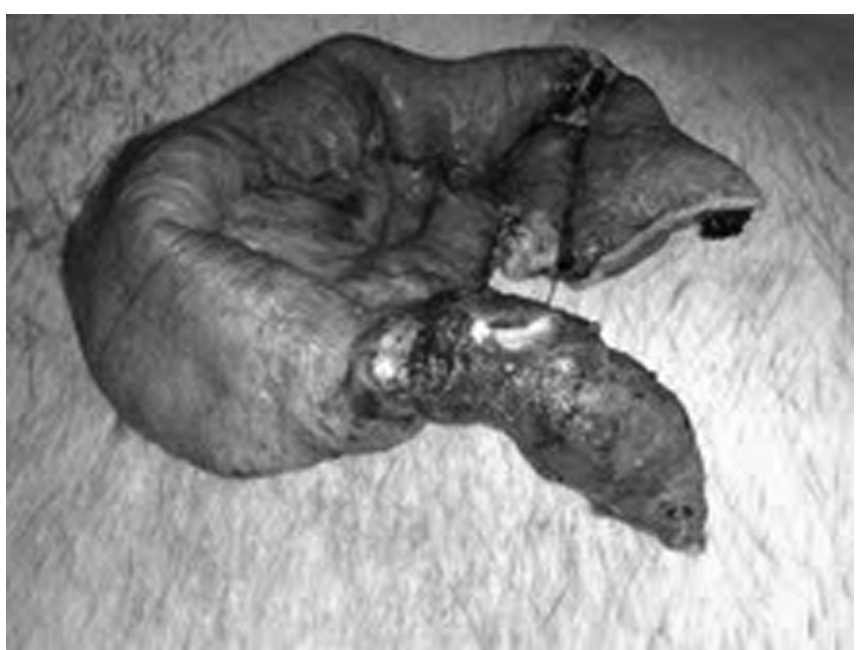

Figure 4. Small bowel perforation distal to an embedded Hemo-lok clip.

complication in 856 operations. With regard to laparoscopic appendectomy, Delibegovic et al ${ }^{16}$ compared titanium clips, endoloops, and Hem-o-lok clips and reported that the mildest inflammatory changes were observed in the titanium clip group followed by the Hem-o-lok group.

However, Liu et $\mathrm{al}^{15}$ showed that the Hem-o-lok clip migrated in the common bile duct after laparoscopic cholecystectomy and common bile duct exploration in 8 of 1600 patients, suggesting that the Hem-o-lok clip may not be a safe method for control of vessels and the cystic duct during laparoscopic cholecystectomy.

There is an interesting report ${ }^{13}$ of a postsurgical smallbowel obstruction caused by a Hem-o-lok clip applied during robot-assisted radical prostatectomy and another report of a clip migrating into the rectum ${ }^{16}$ and presenting as a mass on colonoscopy performed for diverticular disease of the colon.

The manufacturer recommends the use of more than one clip to control the renal artery in procedures other than laparoscopic donor nephrectomy after a contraindication was issued in 2006 in response to reports of death caused by the dislodging of clips from the renal artery. ${ }^{9}$ To achieve a better oncologic outcome in laparoscopic colorectal surgery for malignancy, the surgeon first performs a high division of the IMA in cases of left colon and rectum resection and the ileocolic branch of the superior mesenteric artery in right hemicolectomy. The artery is usually dissected and skeletonized from surrounding mesenteric tissue near its origin and is divided between 1 distal and 2 proximal Hem-o-lok clips. 
We report 2 cases of small-bowel obstruction and perforation related to Hem-o-lok clips. In both cases the 2 proximal clips that were used to ligate the origin of the IMA were dislodged from the artery and found completely embedded in the wall of the bowel, causing full-thickness ischemia, necrosis, and perforation. The clips are normally applied at the origin of the IMA as it branches directly off the abdominal section of the aorta. It is possible therefore that the remnant of the IMA that is clamped is too short for both clips to be applied and, as a result, they slip off the vessel. If this hypothesis is correct, it can be expected to happen in the early postoperative period, leading to torrential bleeding. We believe that it is more likely that, once the small bowel becomes adherent to the clip, the clip will be dislodged by small-bowel peristalsis against resistance. In both cases, the slippage of the clip became evident almost a year later during reoperation when one patient had an emergency laparotomy for bowel obstruction and the other patient had an elective reversal of a Hartmann's procedure. However, if the clips slip off the IMA soon after the operation is performed, then hemorrhage from a major intra-abdominal artery could cause serious complications, including death. Large arteries such as the IMA require the use of large Hem-o-lok clips. The inclusion of too much pulsating tissue within the clip may exert enough pressure to cause it to slip off the artery, but such an event would cause immediate life-threatening complications such as hemorrhage. A Hem-o-lok clip applied on a poorly skeletonized vessel, perhaps encompassing some of the mesenteric tissue and peritoneum, could allow the slow dislodging of the clip, and the process could take long enough to cause obliteration of the lumen of the artery and avoid subsequent bleeding. Histologic evaluation of the small-bowel specimens in both cases did not offer any explanation for the reaction between the nonabsorbable polymeric device and the wall of the bowel. It revealed, however, marked submucosal edema at the indented area of the bowel next to the clip attachment and vascular congestion with abrupt loss of muscularis propria, accompanied by perforation and fibrinopurulent serositis. There was no evidence of dysplasia or malignancy. The features were those of localized ischemia and perforation secondary to extrinsic compression.

\section{CONCLUSION}

When properly applied, Hem-o-lok clips are a safe method of ligating the major mesenteric vessels in elective laparoscopic colorectal procedures. Migration of these clips into the adjacent small bowel, although rare, may cause serious postoperative complications, such as bowel obstruction and perforation, with significant associated morbidity.

It is therefore our recommendation, based on our experience with the Hem-o-lok clip, that the device not be used for the ligation of major mesenteric vessels during laparoscopic colorectal procedures. Large titanium clips or other vascular stapling devices should be used instead.

General and colorectal surgeons should be aware of the reports of Hem-o-lok-related complications when deciding on which mechanical device to use for proximal vascular control during laparoscopic surgery and should promptly report any event that could cause an adverse outcome.

In view of the several reports of Hem-o-lok-related postoperative complications, ${ }^{13-15,17}$ we recommend that large randomized studies be undertaken to determine the effectiveness and safety of these clips and that retroperitoneal operations such as renal transplantation be included in the analysis.

Finally, health care professionals should be familiar with their institutions' current protocols for reporting medical device failures and adverse events. These processes are regulated and legislated by the FDA in the United States through the Medical Device Reporting mechanism and its counterpart in the United Kingdom, the Medicines and Healthcare Products Regulatory Agency, an executive government agency controlled by the Department of Health.

\section{References:}

1. Aminian A, Khorgami Z. Hem-o-lok clip is safe in minimally invasive general surgery: a single center experience and review of data from Food and Drug Administration. J Minim Invas Surg Sci. 2012;1:52-57.

2. Ponsky L, Cherullo E, Moinzadeh A, et al. The Hem-o-lok clip is safe for laparoscopic nephrectomy: a multi-institutional review. Urology. 2008;71:593-596.

3. Koluh A, Delibegovic S, Hasukic S, Valjan V, Latic F. Laparoscopic appendectomy in the treatment of acute appendicitis. Med Arch. 2010;64:147-150.

4. Partecke LI, Kessler W, von Bernstorff W, Diedrich S, Heidecke CD, Patrzyk M. Laparoscopic appendectomy using a single polymeric clip to close the appendicular stump. Langenbecks Arch Surg. 2010;395:1077-1082.

5. Feuer G, Hernandez P, Barker J. Surgical technique enhances the efficiency of robotic hysterectomy. Int J Med Robot. 2011;7:1-6.

6. Yi JS, Kwak C, Kim HH, Ku JH. Surgical clip-related complications after radical prostatectomy. Korean J Urol. 2010;51:683-687. 
7. Bignon H, Buela E, Martinez-Ferro M. Which is the best vessel-sealing method for paediatric thoracoscopic lobectomy? J Laparoendosc Adv Surg Tech A. 2010;20:395-398.

8. Weck Hem-o-Lok Ligating Clips Contraindicated for Ligation of Renal Artery During Laparoscopic Living-Donor Nephrectomy: FDA and HRSA Joint Safety Communication. http://www.fda.gov/MedicalDevices/Safety/AlertsandNotices/ ucm 253237.htm.

9. Teleflex Medical. Letter. Contraindication for use of Hem-Olok ligating clips in laparoscopic donor nephrectomy. 2006; Available from: http//www.swissmedic.ch/recalllists_dl/01155/ Vk_20060608_06-e1.pdf.

10. Mora ER, Gali OB, Garin JA, Arango O. Intravesical migration and spontaneous expulsion of a Hem-o-lok polymer ligating clip after laparoscopic radical prostatectomy. Urology. 2010;75: 1317.

11. Tugcu V, Polat H, Ozbay B, Eren GA, Tasci AI. Stone formation from intravesical Hem-o-lok clip migration after laparoscopic radical prostatectomy. J Endourol. 2009;23: 1111-1113.
12. Tunnard GJ, Biyani CS. An unusual complication of a Hemo-lok clip following laparoscopic radical prostatectomy. J Laparoendosc Adv Surg Tech A. 2009;19:649-651.

13. Ghani KR, Hurwitz M, Menon M. Hem-o-lok clip causing small bowel obstruction after robot-assisted radical prostatectomy. Int J Urol. 2012;19:962-963.

14. Hung SF, Chung SD, Wang SM, Jeff Chueh SC, Yu HJ, Lai MK. Bladder migration of Hem-o-lok clips after laparoscopic radical nephroureterectomy and bladder cuff excision. Surg Laparosc Endosc Percutan Tech. 2011;21:e130-e131.

15. Liu Y, Ji B, Wang Y, Wang G. Hem-o-lok clip found in common bile duct after laparoscopic cholecystectomy and common bile duct exploration: a clinical analysis of 8 cases. Int J Med Sci. 2012;9:225-227.

16. Delibegovic S, Iljazovic E, Katica M, Koluh A. Tissue reaction to absorbable endoloop, nonabsorbable titanium staples, and polymer Hem-o-lok clip after laparoscopic appendectomy. JSLS. 2011;15:70-76.

17. Wu SD, Rios RR, Meeks JJ, Nadler RB. Rectal Hem-o-lok clip migration after robot-assisted laparoscopic radical prostatectomy. Can J Urol. 2009;16:4939-4940. 